\title{
Histological validation of carotid plaque characterization by in-vivo T2 mapping in patients with recent cerebrovascular events: preliminary results
}

Luca Biasiolli ${ }^{*}$, Joshua T Chai ${ }^{1}$, Linqing Li ${ }^{2}$, Ashok Handa ${ }^{3}$, Peter Jezzard ${ }^{2}$, Robin Choudhury ${ }^{1}$, Matthew D Robson ${ }^{1}$

From 17th Annual SCMR Scientific Sessions

New Orleans, LA, USA. 16-19 January 2014

\section{Background}

Multicontrast CMR is the conventional method for invivo characterization of carotid plaques. However, its non-quantitative nature and the need for extensive postacquisition interpretation limit its clinical application. We have recently proposed using in-vivo quantitative T2 mapping for atherosclerotic plaque characterization [Biasiolli et al. JCMR 2013, 15:69]. T2 maps have the advantage of providing an absolute physical measure of plaque components that can be standardized among different MR systems and widely adopted in multi-centre studies. This pilot study investigates the agreement between T2 mapping and histology using AHA plaque type classification.

\section{Methods}

CMR: 19 symptomatic patients scheduled for endarterectomy (14 males, $73 \pm 11$ years, range $54-89$ years, IRB approved, written consent obtained) were imaged at $3 \mathrm{~T}$ (Siemens Verio) with a pair of 2-channel phased-array carotid coils (Machnet). In addition to the conventional multicontrast CMR protocol, a novel black-blood multislice DANTE-prepared [Li et al. MRM 2012, 68:14231438] multiecho-spin-echo sequence was used to acquire 5 slices with 14 echoes $(\mathrm{TR}=2000 \mathrm{~ms}$, $\mathrm{TE}=9$ $127 \mathrm{~ms}$ ) in $<4$ minutes (partial Fourier $=5 / 8, \mathrm{FOV}=$ $128 \times 128$, matrix size $=384 \times 384$, slice thickness $=$ $2 \mathrm{~mm}$, slice gap $=2 \mathrm{~mm}$ ). DANTE parameters: flip angle $=8^{\circ}$, number of pulses $=120$, time duration between pulses $=0.5 \mathrm{~ms}, \mathrm{Gz}=18 \mathrm{mT} / \mathrm{m}$ and gradient

${ }^{1}$ Radcliffe Department of Medicine, University of Oxford, Oxford, UK Full list of author information is available at the end of the article duration $=0.4 \mathrm{~ms}$. T2 maps were generated by monoexponential fitting using non-linear least-squares regression. A reviewer (L.B.) classified plaque types following the CMR-modified AHA scheme using T2 maps + TOF only. Histology: Carotid plaques were freshly obtained at the time of endarterectomy, divided at the level of maximal stenosis into two $4 \mathrm{~mm}$-segments and processed for formalin-fixed paraffin-embedded (FFPE) sections and cryosections, respectively. FFPE sections were stained for H\&E, Masson's trichrome, while cryosections were used for Oil-Red-O/adipophilin (foam cells marker) staining to visualize lipid. AHA type of plaque histology was determined by a reviewer (J.T.C.) blinded to the T2 map results. CMR-histology slice location matching was performed for each $4 \mathrm{~mm}$ segments using the carotid bifurcation as the common anatomical landmark and cross-sectional T1-weighted images of the vessel wall.

\section{Results}

6 of the 19 patients scheduled for endarterectomy were excluded due to severe patient motion artefacts on CMR. The table 1 presents the AHA type classification of the remaining 13 plaques using T2 maps + TOF vs.

Table 1 AHA plaque type classification by CMR (T2 maps +TOF) vs. histology

\begin{tabular}{cccc}
\hline Histology & \multicolumn{3}{c}{ CMR (T2 maps + TOF) } \\
\hline IV-V & VI & total \\
\hline IV & 2 & - & 2 \\
\hline$V$ & 3 & 1 & 4 \\
\hline$V I$ & 1 & 6 & 7 \\
\hline total & 6 & 7 & 13 \\
\hline
\end{tabular}




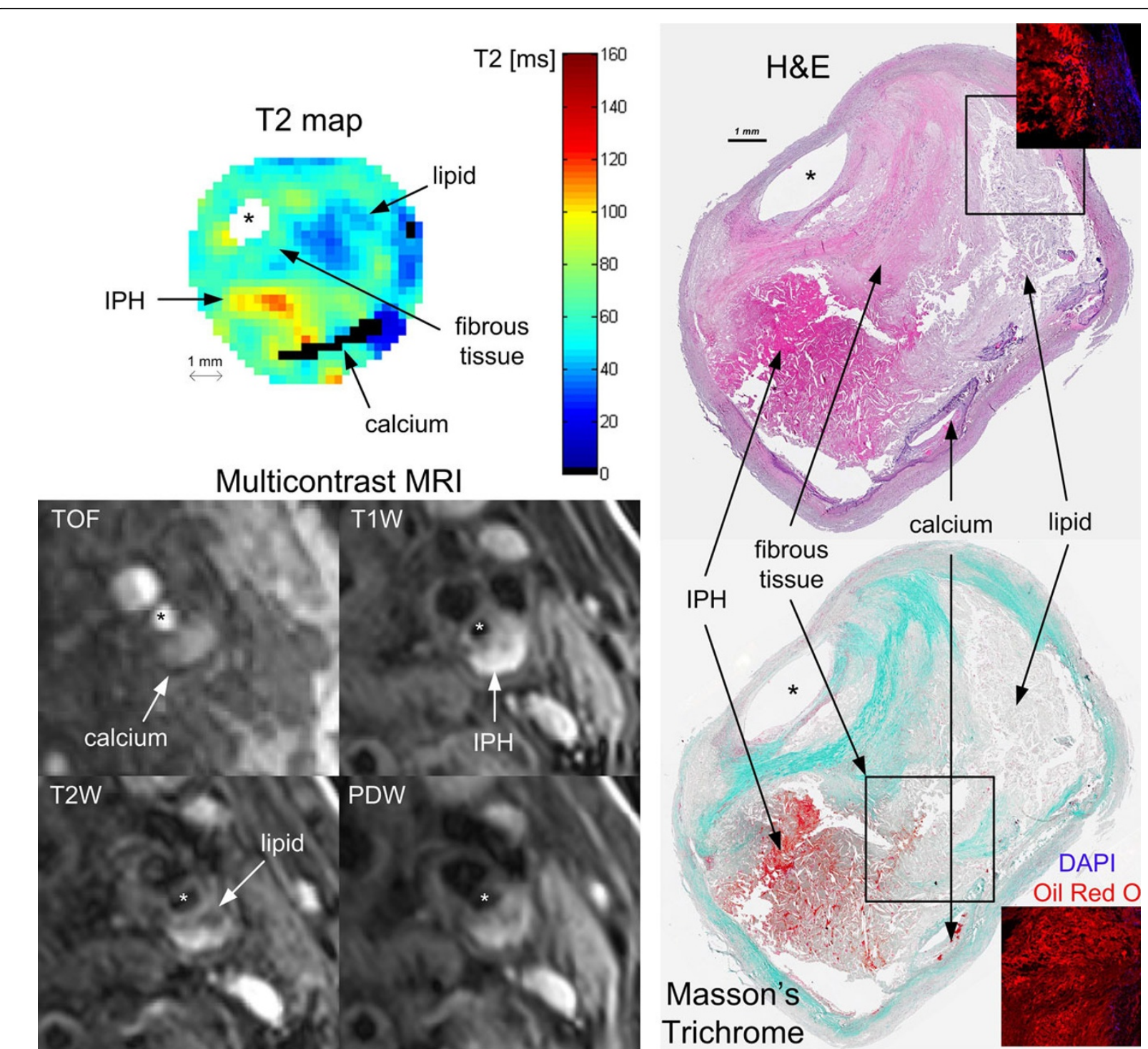

Figure 1 T2 map, multicontrast MRI and histology of a type VI plaque with a large lipid-rich necrotic core (LRNC) separated from the lumen by a thick fibrous cap, clear signs of recent intraplaque-haemorrhage (IPH) and calcification.

histology. The 2 cases of misclassification were due to the difficulty of staging intraplaque haemorrhage (IPH) accurately. T2 maps were able to differentiate lipid-rich necrotic core, fibrous tissue, calcification and recent $\mathrm{IPH}$, as illustrated in the Figure 1 for a type VI plaque.

\section{Conclusions}

These preliminary results show the potential of in-vivo T2 mapping for atherosclerotic plaque characterization. The ability of $\mathrm{T} 2$ maps to discriminate plaque components was confirmed by histology.

\section{Funding}

This study was supported by EPSRC and Oxford Biomedical Research Centre.

\section{Authors' details}

${ }^{1}$ Radcliffe Department of Medicine, University of Oxford, Oxford, UK.

${ }^{2}$ Nuffield Department of Clinical Neurosciences, University of Oxford, Oxford, UK. ${ }^{3}$ Nuffield Department of Surgical Sciences, University of Oxford, Oxford, UK.

Published: 16 January 2014
doi:10.1186/1532-429X-16-S1-P173

Cite this article as: Biasiolli et al:: Histological validation of carotid plaque characterization by in-vivo T2 mapping in patients with recent cerebrovascular events: preliminary results. Journal of Cardiovascular Magnetic Resonance 2014 16(Suppl 1):P173. 